\title{
An Incidentally Discovered Large Left Main Coronary Artery Aneurysm
}

Mohammed Elsadany ${ }^{1,2}$, Jared Selter ${ }^{3}$, Joseph Mattana ${ }^{1,2}$

1. Internal Medicine, St. Vincent's Medical Center, Bridgeport, USA 2. Internal Medicine, Frank H. Netter MD School of Medicine, North Haven, USA 3. Cardiology, St. Vincent's Medical Center, Bridgeport, USA

Corresponding author: Mohammed Elsadany, mohammed.elsadany@hhchealth.org

\begin{abstract}
Left main coronary artery aneurysms (LMCAA) are rare. The most common cause is atherosclerosis followed by congenital malformations. Patients with LMCAA can present with shortness of breath and angina if there is coexisting obstructive coronary artery disease. Here we describe a patient incidentally found to have a 2 $\mathrm{cm}$ aneurysm of the left main coronary artery in the setting of an ST-elevation myocardial infarction (STEMI) and we discuss potential medical and surgical treatment options for this incompletely understood condition.
\end{abstract}

Categories: Cardiology, Internal Medicine

Keywords: left main coronary artery aneurysm, left main aneurysm

\section{Introduction}

Coronary artery aneurysm (CAA) is defined as dilatation of a coronary artery segment to more than 1.5 -fold its normal diameter. It is uncommon with an incidence rate from $0.3 \%$ to $5.3 \%$ [1]. Left main CAA (LMCAA) is rare with a reported incidence rate of $0.1 \%$ [2,3]. Patients with LMCAA can present with ischemic heart disease symptoms if there is coexistent obstructive coronary artery disease within the aneurysm, but others may present with symptoms unrelated to the aneurysm. We describe a patient with a large LMCAA which was detected incidentally during cardiac catheterization after the patient presented with an ST-elevation myocardial infarction (STEMI) secondary to complete occlusion of the mid-right coronary artery.

Received 06/12/2020 Review began 06/27/2020 Review ended 07/06/2020 Published 07/13/2020

\section{(๑) Copyright 2020}

Elsadany et al. This is an open access article distributed under the terms of the Creative Commons Attribution License CC-BY 4.0., which permits unrestricted use, distribution, and reproduction in any medium, provided the original author and source are credited.

\section{Case Presentation}

A 64-year-old woman with a history of hypertension presented to the emergency department with sudden onset mid-sternal chest discomfort. Electrocardiography confirmed an acute inferior wall STEMI and she underwent emergent cardiac catheterization. Angiography showed a $75 \%$ stenosis in the first diagonal and complete occlusion of the mid-right coronary artery which was the cause for the patient's inferior wall STEMI and was successfully revascularized with the placement of a single bare-metal stent. In addition, a 2 $\mathrm{cm}$ saccular aneurysm was noted in the distal left main (LM) coronary artery (Figures 1-2). The LM bifurcation was obscured by the aneurysm and a high-grade stenosis could not be excluded. Intravascular ultrasonography was not performed given the high risk of perforation. Surgical intervention was not pursued during her hospitalization given her recent infarct and equivocal LM findings. Her post-infarct course was uneventful, and she was discharged on a regimen of aspirin, ticagrelor, carvedilol, and atorvastatin. 


\section{Cureus}

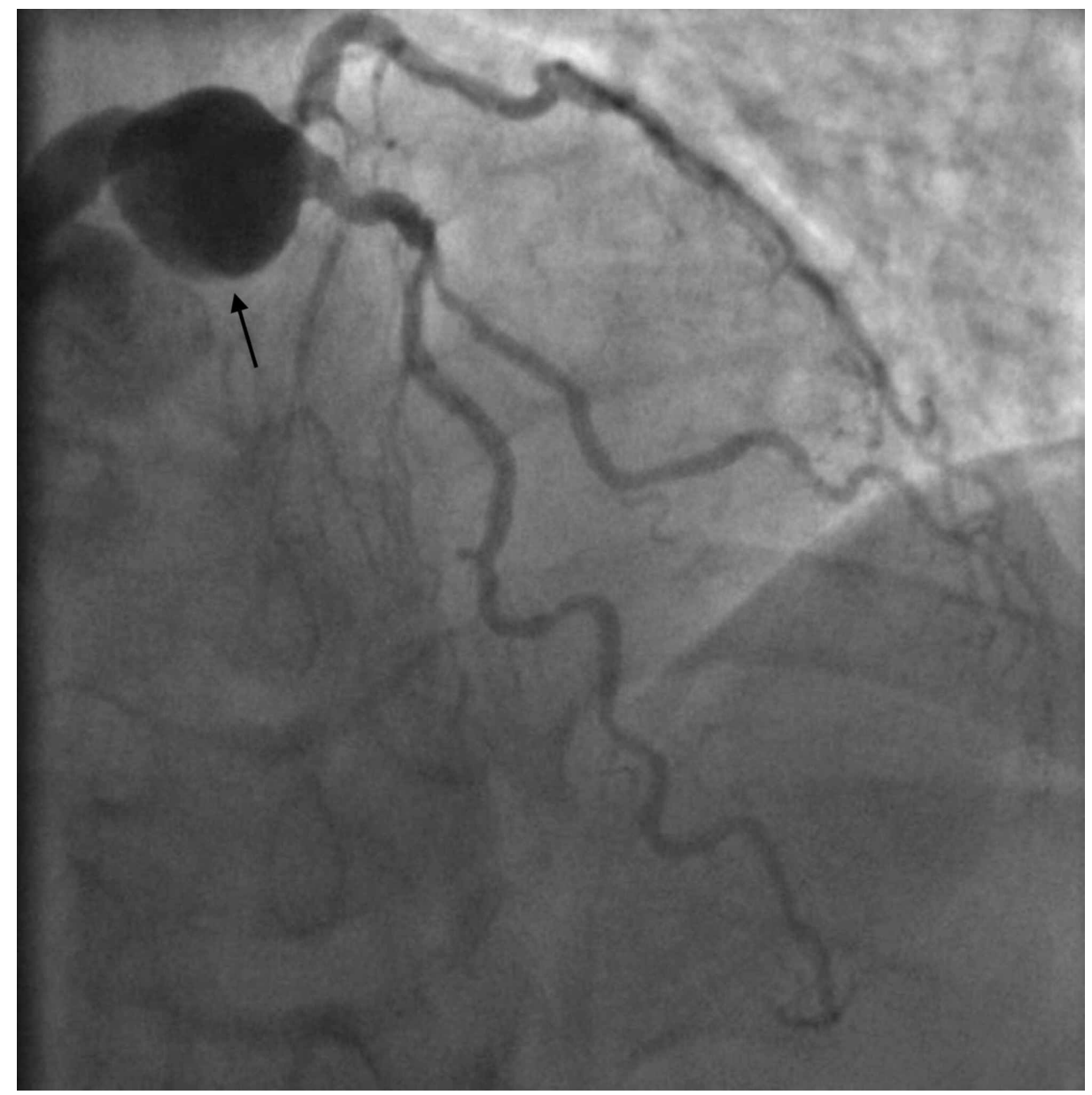

FIGURE 1: Left heart catheterization RAO cranial view showing left main coronary artery aneurysm and $75 \%$ stenosis in the first diagonal.

RAO: right anterior oblique.

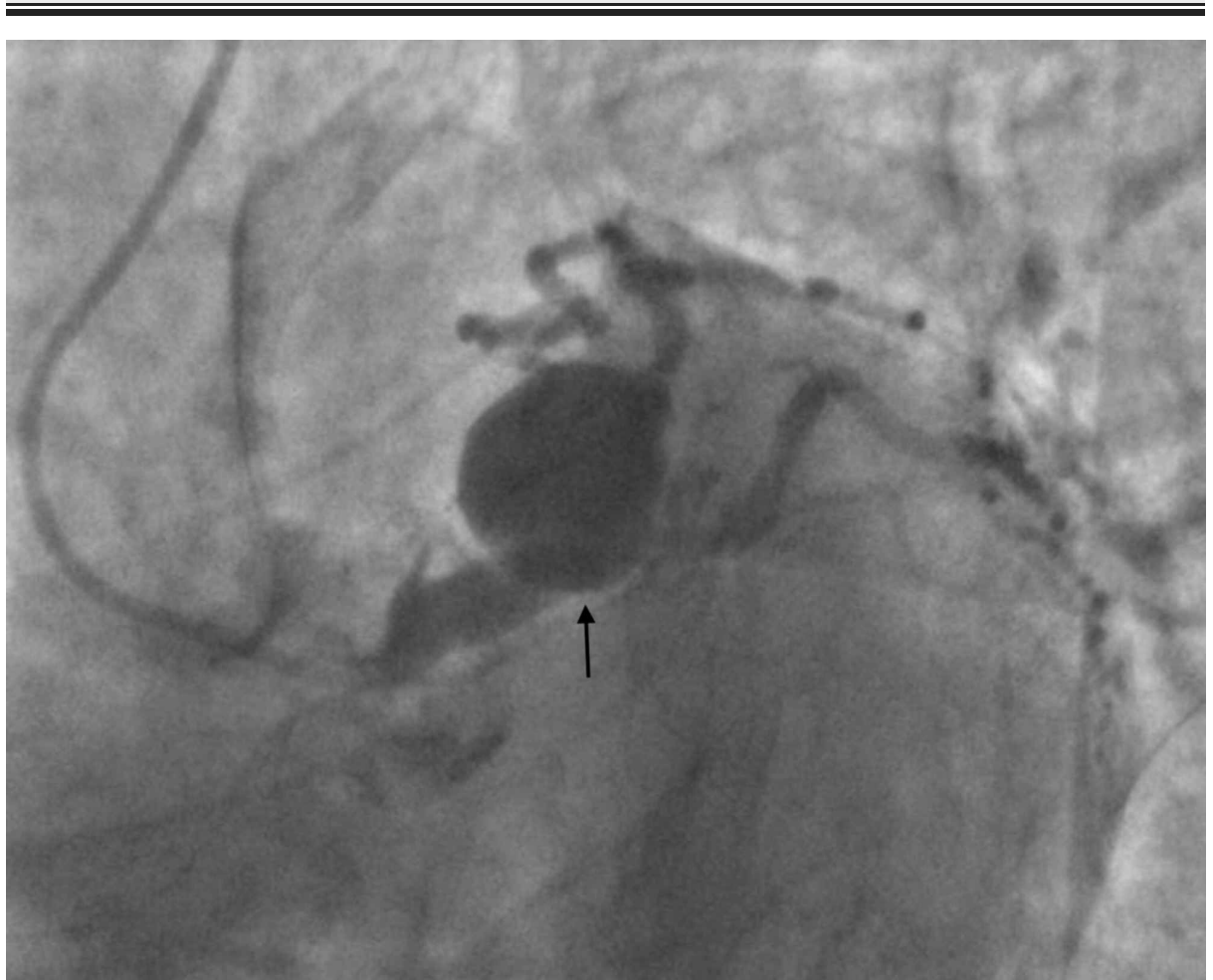




\section{Discussion}

CAA is defined as dilatation of a coronary artery segment to more than 1.5 -fold its normal diameter. CAA is uncommon with an incidence rate from $0.3 \%$ to $5.3 \%$ [1]. Aneurysms can be either focal or diffuse and are classified according to morphology: fusiform or saccular. The most commonly affected coronary artery is the right (40\%-87\% of aneurysms). The involvement of the LM, as found in our patient, is rare with an incidence of only $0.1 \%$ [2,3]. The most common cause of CAA in adults is atherosclerosis ( $50 \%$ of cases) followed by congenital malformations (20\%-30\% of cases) [2]. Other causes include vasculitis, Marfan syndrome, traumatic injury, and idiopathic. Coronary angiography is the gold standard for diagnosis [4].

Patients with CAA are usually asymptomatic, though patients can present with shortness of breath and angina when obstructive coronary artery disease exists within the aneurysm. Furthermore, abnormal flow within a CAA may predispose to thrombus formation and distal embolization leading to myocardial infarction without the presence of obstructive coronary artery disease [5]. Our patient's STEMI was unrelated to her CAA.

The treatment of CAA is not well established due to the rarity of the condition. Treatment recommendations are based on case reports and expert consensus. Surgical intervention is indicated in patients with coexistent obstructive coronary artery disease within the aneurysm if myocardial infarction develops secondary to embolic events from the aneurysm, or in cases of progressive CAA enlargement documented by serial coronary imaging. Surgical interventions in LMCAA are usually performed by proximal and distal ligation of the aneurysm with bypass grafts to the left anterior descending and left circumflex coronary arteries. For medical treatment, current reports recommend starting antiplatelet and anticoagulation therapy to prevent thrombus formation within the aneurysm [6]. There is considerable uncertainty as to the optimal approach in a patient such as ours, given her need for dual antiplatelet therapy and increased risk of bleeding.

\section{Conclusions}

CAA, especially LMCAA, is uncommon, with atherosclerosis being the most common etiology. Given its rarity, treatment of LMCAA is not well established, though surgical intervention is recommended if obstructive coronary artery disease is present within the aneurysm, if distal embolization with myocardial infarction occurs or if the aneurysm progressively enlarges. Recommended medical treatment includes antiplatelet and anticoagulation therapy to prevent thrombus formation within the aneurysm. Further study of this incompletely understood condition will hopefully lead to better guidance for optimal management of the scenario our patient presented with.

\section{Additional Information \\ Disclosures}

Human subjects: Consent was obtained by all participants in this study. Conflicts of interest: In compliance with the ICMJE uniform disclosure form, all authors declare the following: Payment/services info: All authors have declared that no financial support was received from any organization for the submitted work. Financial relationships: All authors have declared that they have no financial relationships at present or within the previous three years with any organizations that might have an interest in the submitted work. Other relationships: All authors have declared that there are no other relationships or activities that could appear to have influenced the submitted work.

\section{References}

1. Hartnell GG, Parnell BM, Pridie RB: Coronary artery ectasia. Its prevalence and clinical significance in 4993 patients. Br Heart J. 1985, 54:392-5. 10.1136/hrt.54.4.392

2. Cohen P, O'Gara PT: Coronary artery aneurysms: a review of the natural history, pathophysiology, and management. Cardiol Rev. 2008, 16:301-4. 10.1097/CRD.0b013e3181852659

3. Topaz O, DiSciascio G, Cowley MJ, et al.: Angiographic features of left main coronary artery aneurysms . Am J Cardiol. 1991, 67:1139-42. 10.1016/0002-9149(91)90881-k

4. Sokmen G, Tuncer C, Sokmen A, Suner A: Clinical and angiographic features of large left main coronary artery aneurysms. Int J Cardiol. 2008, 123:79-83. 10.1016/j.ijcard.2007.01.054

5. Rath S, Har-Zahav Y, Battler A, et al.: Fate of nonobstructive aneurysmatic coronary artery disease: angiographic and clinical follow-up report. Am Heart J. 1985, 109:785-91. 10.1016/0002-8703(85)90639-8

6. Lima B, Varma S, Lowe JE: Nonsurgical management of left main coronary artery aneurysms: report of 2 cases and review of the literature. Tex Heart Inst J. 2006, 33:376-379. 